\title{
Clinical Practice Guidelines for Hepatocellular Carcinoma Differ between Japan, United States, and Europe
}

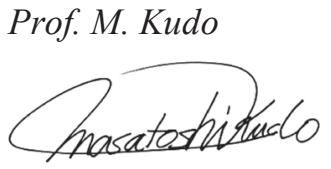

Editor Liver Cancer

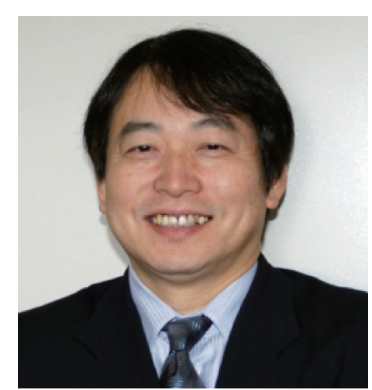

\section{Introduction}

Hepatocellular carcinoma (HCC) is a malignant tumor that is the fifth most common type of cancer and the third leading cause of cancer-related death globally. Although HCC was once thought to be a special type of cancer prevalent only in Southeast Asia and Africa, it has rapidly become more common in other regions, particularly Europe and the United States, which has led to greater interest in the diagnosis and treatment of HCC worldwide. Consequently, the American Association for the Study of Liver Diseases (AASLD) [1] and the European Association for the Study of the Liver and the European Organisation for Research and Treatment of Cancer (EASL-EORTC) [2] have published clinical practice guidelines for liver cancer. In Japan, the first edition of evidence-based clinical practice guidelines was published in 2005 [3, 4], followed by a revised edition in 2009 and a third revision in 2013 [5, 6]. In addition, 1 or 2 years after the publication of each revised edition of the evidence-based treatment guidelines, The Japan Society of Hepatology (JSH) has also published consensusbased clinical practice manuals for HCC. The first edition was published in 2007 and the second edition in 2010 [7, 8]. A third edition will be published in 2015. In this article, differences between the aforementioned European and American clinical practice guidelines and Japanese clinical practice guidelines will be discussed. Specifically, as these guidelines primarily consist of both a surveillance and diagnostic algorithm and a treatment algorithm, each component of the algorithms will be explained in detail.

\section{Surveillance and Diagnostic Algorithms}

The AASLD diagnostic algorithm (fig. 1) and the EASL-EORTC diagnostic algorithm (fig. 2) are basically the same; the AASLD and EASL-EORTC surveillance and diagnostic algo- 


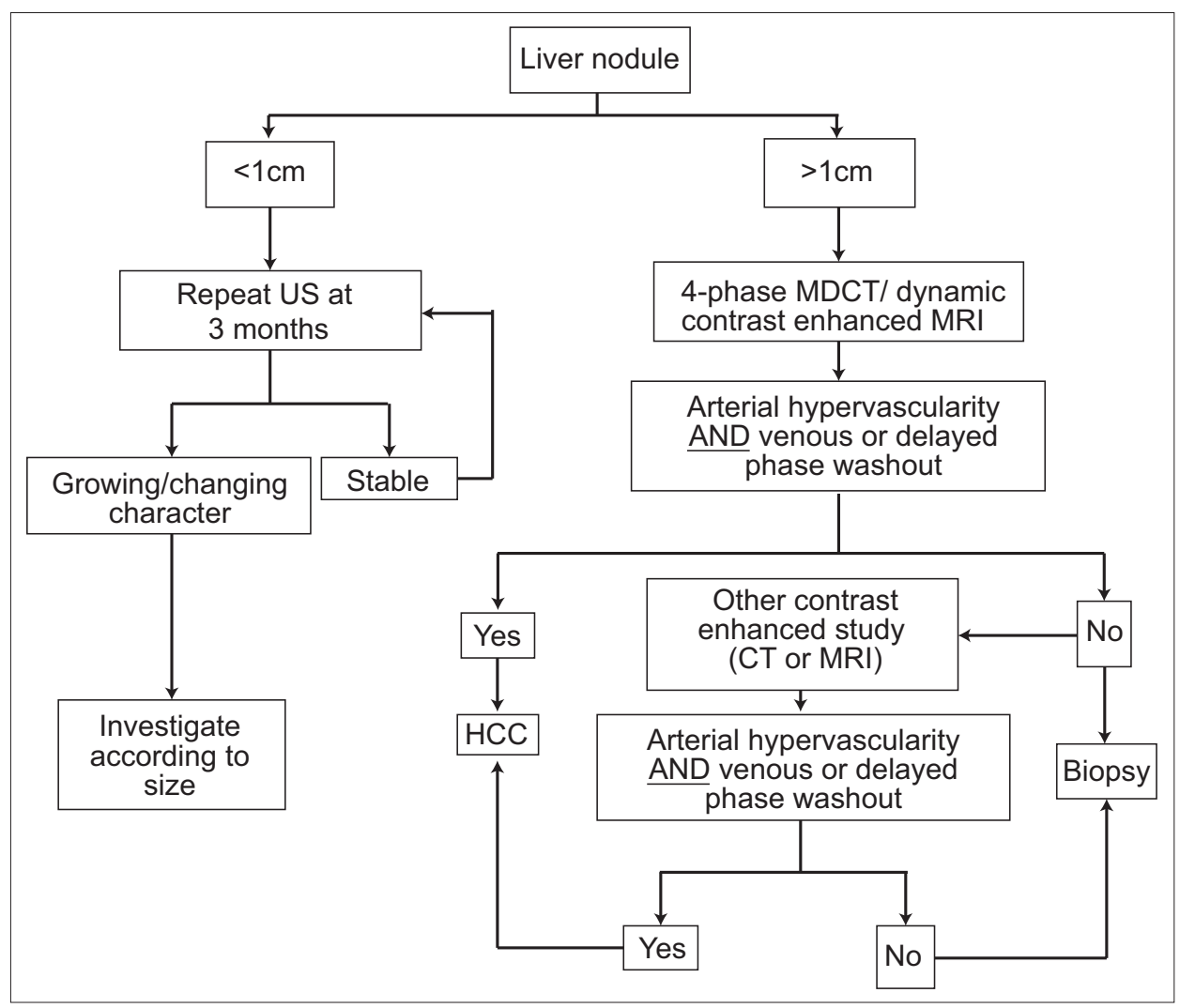

Fig. 1. AASLD practice guidelines for HCC surveillance and diagnosis [1]. MDCT=multidetector CT; US=ultrasound. Modified with permission from Bruix J et al. [1].

rithms differ from the JSH surveillance algorithm in that they do not make use of tumor markers such as alpha-fetoprotein (AFP) and recommend surveillance with ultrasound alone. Essentially, for the AASLD and EASL-EORTC surveillance and diagnostic algorithms, only cirrhotic patients are considered candidates for surveillance, and surveillance is performed with ultrasound every 6 months. If a nodule $\leq 1 \mathrm{~cm}$ is found, ultrasound examinations are performed every 3 months, and dynamic computed tomography (CT) or magnetic resonance imaging (MRI) scans are not performed unless the nodule exceeds $1 \mathrm{~cm}$. If arterial enhancement and venous equilibrium phase washout are observed with either modality, HCC can be diagnosed noninvasively. If a diagnosis cannot be made by CT or MRI, HCC can be diagnosed if this imaging hallmark is observed in another dynamic study. Liver biopsy is recommended only when these imaging features are not observed.

The algorithm in the EASL-EORTC guidelines differs only in some minor respects from the AASLD algorithm. When a tumor $\leq 1 \mathrm{~cm}$ is detected, both algorithms propose that CT and MRI examinations should not be performed, but the AASLD algorithm recommends an ultrasound examination every 3 months, whereas the EASL algorithm recommends an ultrasound examination every 4 months. In addition, although the EASL guidelines present different diagnostic algorithms for 1 - to 2 - $\mathrm{cm}$ nodules and nodules $>2 \mathrm{~cm}$, this distinction is essentially meaningless, and the EASL algorithm is largely the same as the AASLD algorithm. As such, it should not really be necessary to have separate approaches for 1- to 2-cm nodules and nodules $>2 \mathrm{~cm}$. Specifically, the EASL guidelines state that typical features of HCC must be observed with both modalities (CT and MRI) to diagnose 1- to 2-cm nodules in institutions other than centers of excellence, whereas the clear appearance of typical features 


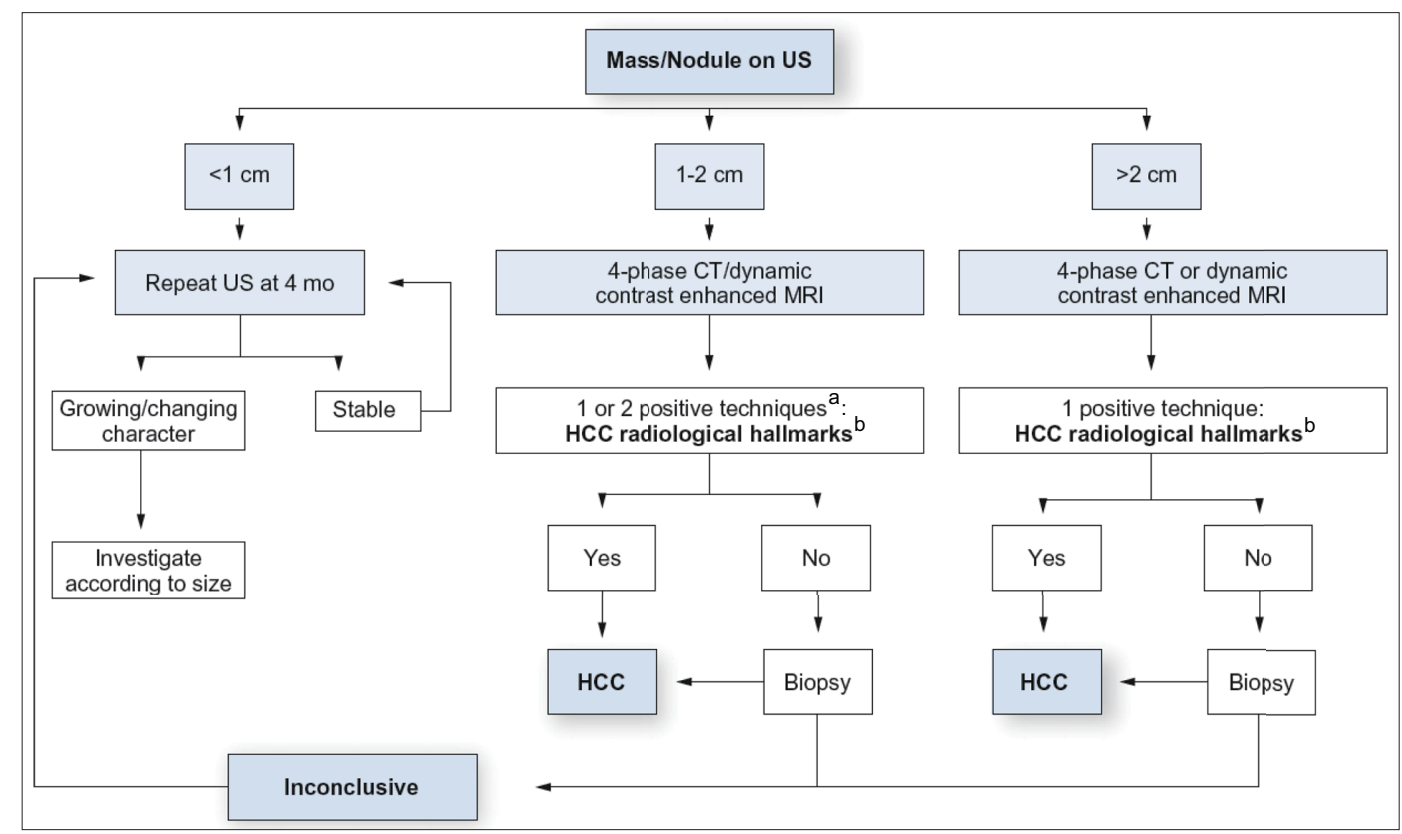

Fig. 2. EASL-EORTC practice guidelines for HCC surveillance and diagnosis [2].

${ }^{a}$ One imaging technique only recommended in centers of excellence with high-end radiological equipment. ${ }^{b} \mathrm{HCC}$ radiological hallmarks: arterial hypervascularity and venous/late phase washout.

Modified with permission from EASL-EORTC. [2].

of HCC on only one dynamic study is sufficient to diagnose nodules $>2 \mathrm{~cm}$. This essentially means that a single modality is sufficient for either size of nodule, so there is no need to deliberately differentiate the two sizes of nodules. The EASL and AASLD algorithms have identical recommendations for cirrhotic patients: they both recommend only an ultrasound examination every 6 months and do not recommend the use of tumor markers.

The Japanese evidence-based clinical practice guidelines for HCC (fig. 3) deliberately divide patients into an extremely high-risk group (hepatitis B or C cirrhosis) and a high-risk group (patients with chronic hepatitis B, chronic hepatitis $\mathrm{C}$, or non-viral cirrhosis) and recommend an ultrasound examination every 3-4 months along with measurement of three tumor markers [AFP, proteins induced by vitamin $\mathrm{K}$ absence (PIVKA-II), and AFP-L3] [9, 10] every 3-4 months for the extremely high-risk group. They also recommend CT and MRI examinations every 6-12 months as an optional screening method. For the high-risk group, they recommend an ultrasound examination every 6 months and measurement of three tumor markers every 6 months.

The Japanese diagnostic algorithm differs from the European and American algorithms in that it recommends gadolinium-ethoxybenzyl-diethylenetriamine pentaacetic acid-enhanced MRI (EOB-MRI), contrast-enhanced ultrasound (CEUS), or CT angiography as an optional imaging test when a nodule shows early contrast enhancement without late washout and is $\geq 1 \mathrm{~cm}$; if a diagnosis is not confirmed, then liver tumor biopsy is recommended. In other words, The Japanese algorithm takes a more rigorous approach to noninvasive diagnosis. If there is no early contrast enhancement and the tumor diameter is $>1.5 \mathrm{~cm}$, the same optional tests can be performed; however, if it is $\leq 1.5 \mathrm{~cm}$, the patient should be followed every 3 months and another dynamic CT or MRI performed if the tumor enlarges or tumor marker levels increase. If the tumor size is unchanged or regresses, the algorithm recommends the regular surveillance schedule be resumed. 


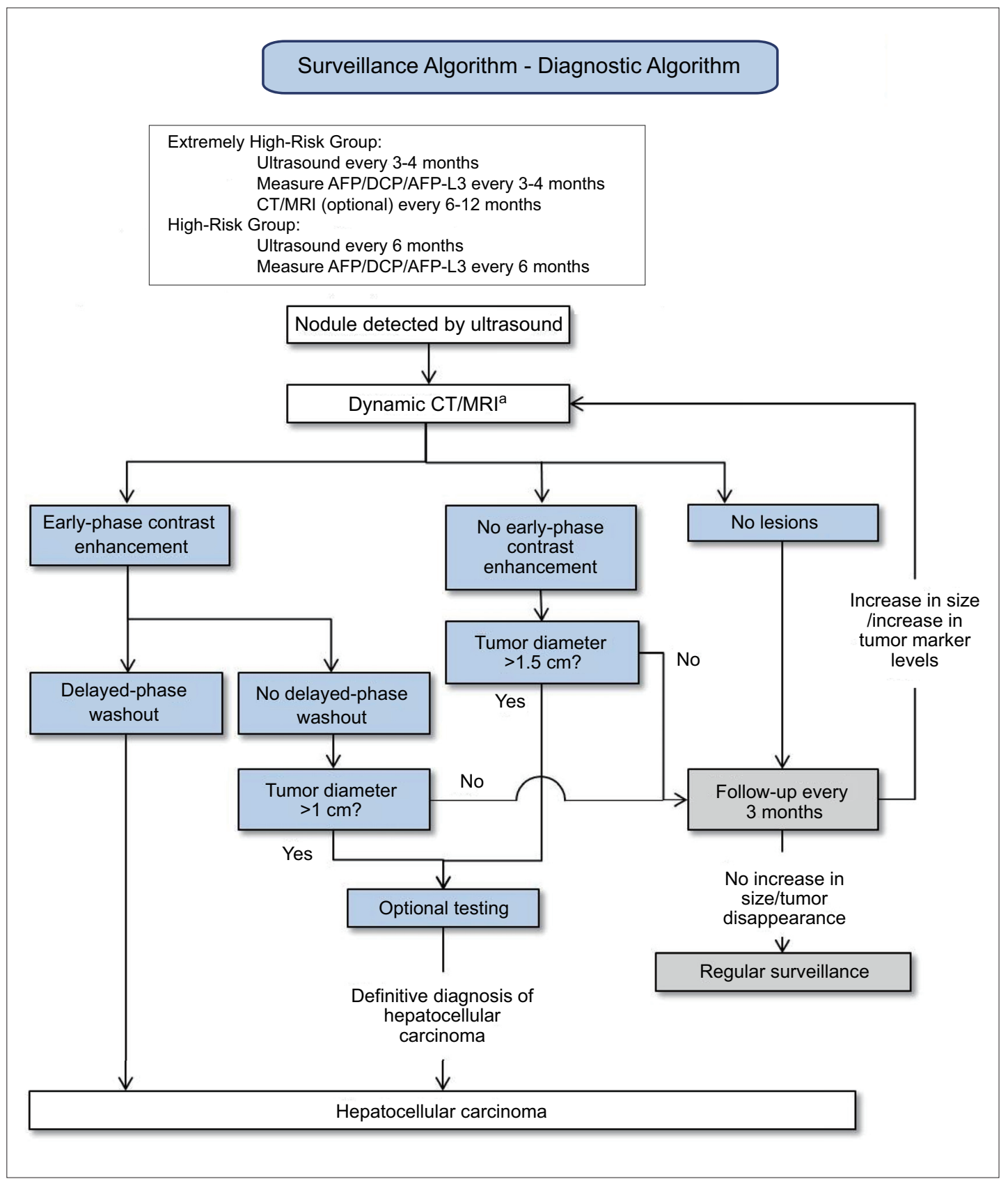

Fig. 3. Japanese evidence-based clinical practice guidelines for HCC: surveillance and diagnostic algorithm [6]. AFP=alpha-fetoprotein; DCP=des-gamma carboxyprothrombin (also known as PIVKA-II). Optional testing=EOB-MRI, CEUS, CT angiography or tumor biopsy.

${ }^{\mathrm{a}} \mathrm{CT} / \mathrm{MRI}$ are used for some patients even if the nodule(s) are not visualized using ultrasound because of poor visualization capability. Contrast-enhanced ultrasound may be considered for patients with renal impairment and/or allergies to contrast media of CT/MRI. Modified with permission from Kokudo N et al. [6].

As in the Japanese evidence-based guidelines, the consensus-based clinical practice guidelines for liver cancer (fig. 4) use identical definitions for the extremely high-risk group and high-risk group. They are also similar to the evidence-based guidelines in that they recommend surveillance with dynamic CT or MRI every 6-12 months for cirrhotic patients be- 


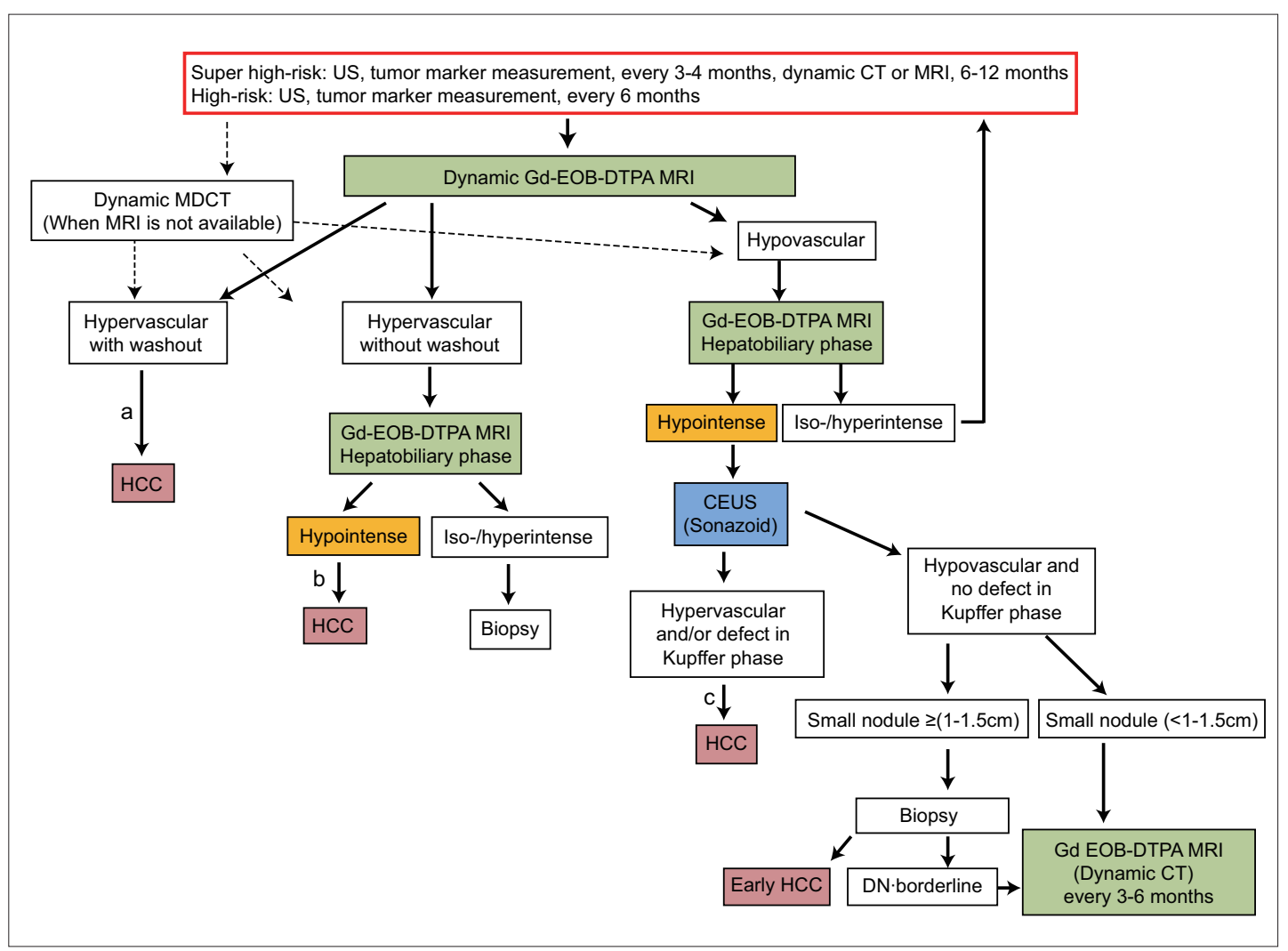

Fig. 4. Consensus-based surveillance and diagnostic algorithm for HCC (Proposed by Liver Cancer Study Group of Japan 2014 [19]). Gd-EOB-DTPA=gadolinium-ethoxybenzyl-diethylenetriamine pentaacetic acid-enhanced; $\mathrm{DN}=$ dysplastic nodule. $\mathrm{SPIO}=$ superparamagnetic iron oxide; $\mathrm{CTAP}=\mathrm{CT}$ during arterial portography; CTHA=CT during hepatic arteriography. Optional examinations such as SPIO MRI, CTAP, CTHA and highly sensitive tumor marker measurement are recommended in difficult to diagnose cases.

${ }^{a}$ Cavernous hemangioma may show hypointensity on equilibrium (transitional) phase of dynamic GdEOB-DTPA MRI (pseudo-washout). Cavernous hemangioma should be excluded by other sequences of MRI and/or other imaging modalities. ${ }^{b}$ Cavernous hemangioma usually shows hypointensity on hepatobiliary phase of Gd-EOB-DTPA MRI. Cavernous hemangioma should be excluded by other sequences of MRI and/ or other imaging modalities. 'Biopsy may be considered for confirmation.

Modified with permission from Kudo M et al. [19].

cause nodules may not be detected on ultrasound alone. However, the consensus-based algorithm differs in that it recommends EOB-MRI, which has higher detection sensitivity than CT, as the first-line modality for surveillance every 6-12 months. This algorithm classifies nodules as having one of three patterns: arterial hypervascularity and late washout, hypervascularity but no late washout, or arterial hypovascularity. Hypervascular nodules with washout can be diagnosed as HCC by imaging alone. Hypervascular nodules without washout can be diagnosed as HCC if they are hypointense in the hepatobiliary phase of EOB-MRI. Biopsy is recommended if the nodules are isointense or hyperintense in the hepatobiliary phase.

Nodules that are hypovascular on EOB-MRI have strong malignant potential if they are hypointense in the hepatobiliary phase and therefore, in these cases, Sonazoid CEUS is performed. If the nodule is hypervascular on Sonazoid CEUS or there is a defect in the Kupffer phase, it is definitively diagnosed as HCC, although biopsy can be performed as an optional test. This is because such cases may include not only well-differentiated HCC but also moderately and poorly differentiated HCC, which could affect the treatment options. If the nodule is 


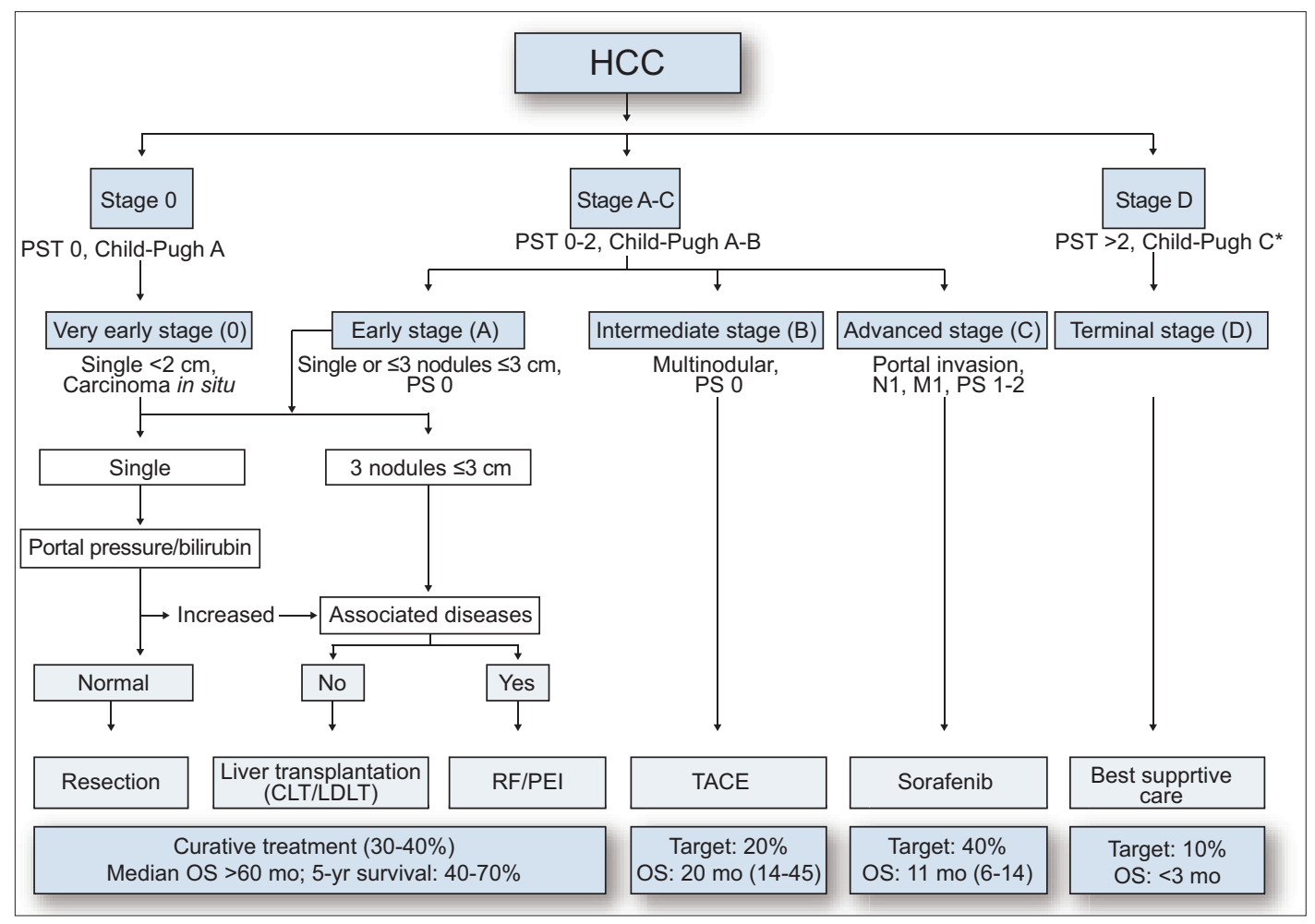

Fig.5. AASLD/EASL-EORTC treatmentalgorithm for $\operatorname{HCC}(1,2)$. PST=performance status; $C L T=$ cadaveric liver transplantation; LDLT=living donor liver transplantation; RF/PEI=radiofrequency ablation/percutaneous ethanol injection; OS=overall survival; mo=months; Target $20 \%$ means expected target population is $20 \%$. The numbers in parentheses after the overall survival in months indicate the OS range of reported series. Modified with permission from Bruix J et al. and EASL-EORTC.[1,2].

neither hypovascular on Sonazoid CEUS nor shows a defect in the Kupffer phase, biopsy is recommended if the nodule is $1-1.5 \mathrm{~cm}$ or larger in order to differentiate between early HCC and a dysplastic nodule [11]. Even small nodules 1-1.5 cm or smaller have a strong potential to become hypervascular if they are hypointense in the hepatobiliary phase of EOB-MRI, so intensive follow up with EOB-MRI is recommended every 3-6 months. A fundamental principle of these consensus-based guidelines is that for institutions that cannot perform EOB-MRI so frequently, it is acceptable to substitute dynamic CT for both surveillance and follow up.

To summarize, the Japanese guidelines differ completely from the European and American guidelines in that they propose a diagnosis be made on the basis of very sophisticated surveillance, and this aspect makes them superior.

\section{Treatment Algorithms}

The treatment algorithms in the AASLD and EASL-EORTC guidelines are identical (fig. 5). Basically, they use the Barcelona-Clinic Liver Cancer (BCLC) staging system and define very early stage cancer $(<2 \mathrm{~cm}$, single nodule, Child-Pugh $A)$ as stage 0 and cases involving single nodules or $\leq 3$ nodules of $\leq 3 \mathrm{~cm}$ as early stage (stage A) HCC. They define multinodular HCC as intermediate stage (B), cases involving vascular invasion or extrahepatic spread as advanced stage $(\mathrm{C})$, and cases where the patient has Child-Pugh $\mathrm{C}$ cirrhosis or a perfor- 


\section{Treatment Algorithm}

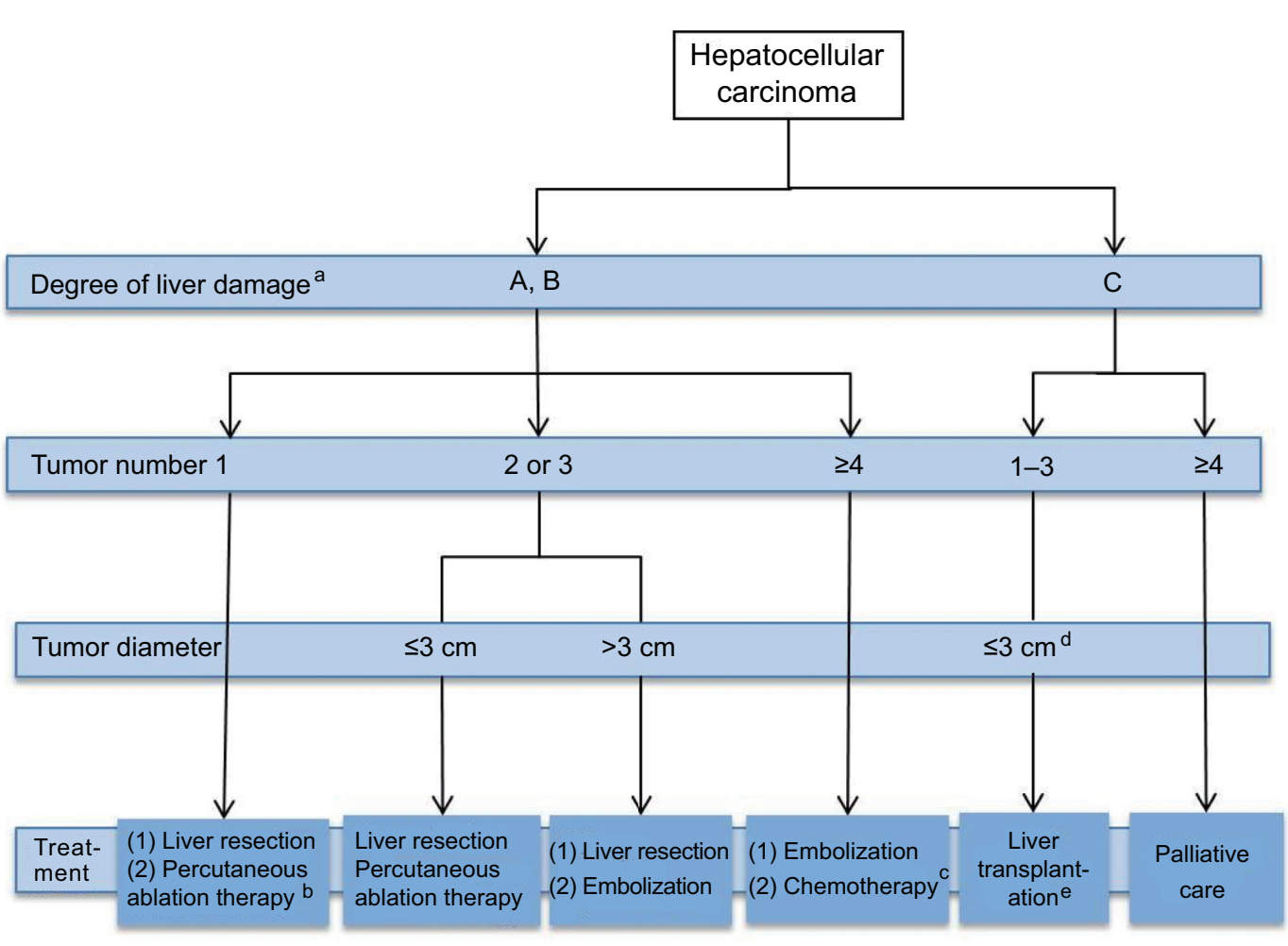

- At times, liver resection, chemotherapy, and embolization therapy may be selected for patients with Child-Pugh class A liver damage along with vascular invasion

- Chemotherapy is recommended for patients with Child-Pugh class A disease with extrahepatic metastases

Fig. 6. Japanese evidence-based clinical practice guidelines for hepatocellular carcinoma: treatment algorithm [6].

aThe Child-Pugh classification may also be used when non-surgical treatment is considered. ${ }^{\mathrm{b}} \mathrm{Can}$ be selected for tumors with a diameter of $\leq 3 \mathrm{~cm}$. ${ }^{\mathrm{c} O r a l}$ administration and/or hepatic arterial infusion are

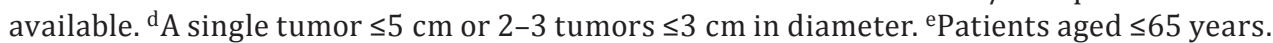

Modified with permission from Kokudo $\mathrm{N}$ et al. [6].

mance status $\geq 2$ as terminal stage (D), with a different treatment option recommended for each stage. They recommend resection [12] and ablation [13] or transplantation [14] for very early stage and early stage HCC, transcatheter arterial chemoembolization (TACE) [15] for intermediate-stage HCC, sorafenib for advanced-stage HCC [16], and best supportive care for terminal-stage HCC.

In contrast, the Japanese evidence-based clinical practice guidelines (fig. 6) recommend resection as the first-line treatment in patients with Child-Pugh A or B liver function with a single tumor, with ablation as an alternative when the tumor is $\leq 3 \mathrm{~cm}$. Resection and ablation are both options for patients with 2-3 tumors that are $\leq 3 \mathrm{~cm}$, and resection is considered the first-line treatment and embolization the second-line treatment for patients with 2-3 tumors $>3 \mathrm{~cm}$. Embolization is considered the first-line treatment and hepatic arterial infusion che- 
motherapy (HAIC) [17] the second-line treatment for patients with $\geq 4$ tumors. It should be noted that chemotherapy includes oral drugs (sorafenib) and HAIC. Liver transplantation is recommended for patients with Child-Pugh $\mathrm{C}$ cirrhosis who meet the Milan criteria, but the maximum age is generally 65 years. Palliative care is recommended for patients who do not meet the Milan criteria.

The consensus-based treatment algorithm (fig. 7), in contrast, establishes different treatment options based on five factors: extrahepatic spread, liver function, vascular invasion, tumor number, and tumor size. This algorithm differs from the European and American algorithms in the following aspects. First, it recommends intensive follow up or ablation for hypovascular pathologically early HCC [18]. Also, it recommends a combination of TACE and ablation for patients with $\leq 3$ tumors that are $>3 \mathrm{~cm}$ in size. Moreover, it considers resection and ablation to be indicated for patients with multiple nodules $(\geq 4)$ when possible, if used in combination with TACE. It also recommends HAIC for patients with multiple nodules. For Child-Pugh A/B patients with vascular invasion, it recommends HAIC in addition to sorafenib $[17,19]$. If the vascular invasion is minor (e.g., Vp1 or Vp2), TACE or resection is also recommended. Finally, locoregional therapy performed in Japan is believed to confer a survival benefit for HCC patients with Child-Pugh C cirrhosis who meet the Milan criteria and have a Child-Pugh score of 10 or 11 [20-25]. Alternatives such as ablation and TACE are recommended if liver transplantation is not possible.

\section{Summary}

The main differences between the surveillance and diagnostic algorithms of Europe and USA and those of Japan can be summarized as follows.

1) Whereas the European and American guidelines propose a surveillance interval of 6 months regardless of whether the patient has cirrhosis, the Japanese guidelines propose a shorter interval of 3-4 months for cirrhotic patients. Because of this, more cases of small HCCs are detected in Japan compared with Europe and America.

2) The surveillance methods also differ. Whereas the three tumor markers AFP, PIVKA-II, and AFP-L3 are used in addition to ultrasound in Japan, not even AFP is recommended in Europe and America, and PIVKA-II and AFP-L3 are not recommended because these tests are not routinely available outside Japan.

3) The Japanese guidelines recommend EOB-MRI or dynamic CT be performed every 6-12 months for surveillance for cirrhotic patients.

4) The European and American diagnostic guidelines recommend prompt biopsy when typical features of liver cancer are not observed, whereas the Japanese guidelines recommend more sophisticated imaging, such as hepatobiliary-phase EOB-MRI or Sonazoid CEUS.

5) Another major difference is that even hypovascular pathologically early HCC is diagnosed with EOB-MRI or biopsy in Japan.

6) The Japanese guidelines propose that when HCC cannot be detected with ultrasound, screening with EOB-MRI should be done every 6-12 months. This has enabled the detection of a large number of very small HCCs, including hypovascular pathologically early HCC.

The Japanese treatment algorithm's are different and considered to be superior to the European and American treatment algorithms for the following reasons.

1) Consensus-based treatment algorithm provides a treatment option for hypovascular pathologically early HCC.

2) When used in combination with TACE, ablation is still indicated as a treatment for patients with $\leq 3$ nodules that are $>3 \mathrm{~cm}$. 


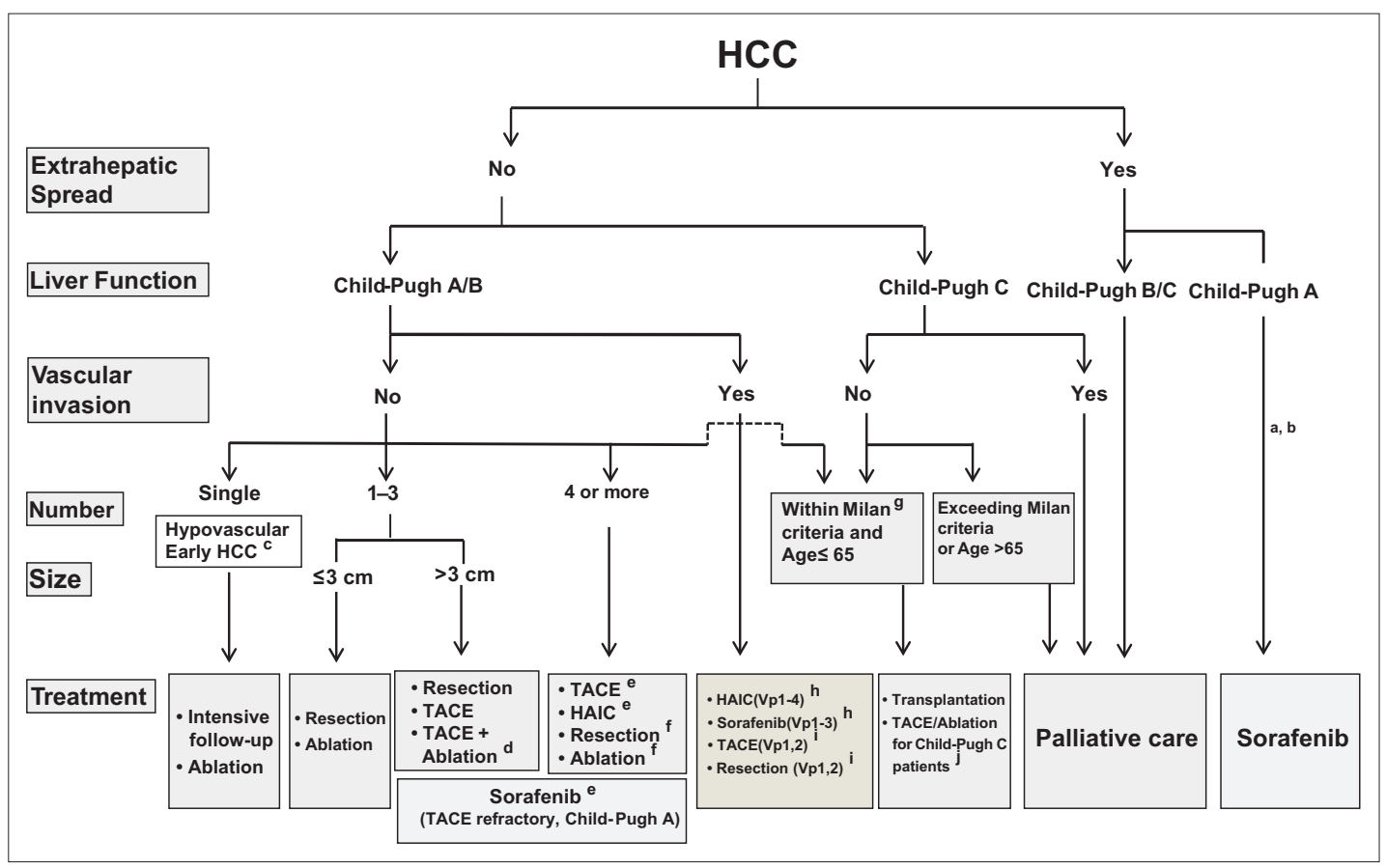

Fig. 7. JSH/Liver Cancer Study Group of Japan consensus-based treatment algorithm for HCC revised in 2014 [19]. HAIC=hepatic arterial infusion chemotherapy.

aTreatment should be performed as if extrahepatic spread is negative, when extrahepatic spread is not regarded as a prognostic factor. bSorafenib is the first choice of treatment in this setting as a standard of care. 'Intensive follow-up observation is recommended for hypovascular nodules by the Japanese Evidence-Based Clinical Practice Guidelines. However, local ablation therapy is frequently performed in the following cases: 1) when the nodule is diagnosed pathologically as early HCC, 2) when the nodules show decreased uptake on hepatobiliary-phase Gd-EOB-MRI, 3) when the nodules show decreased portal flow by CTAP or 4) decreased uptake is shown on Kupffer phase of Sonazoid-enhanced US, since these nodules are known to frequently progress to typical hypervascular HCC. 'Even for HCC nodules exceeding $3 \mathrm{~cm}$ in diameter, combination therapy of TACE and ablation is frequently performed when resection is not indicated. ${ }^{\text {TTACE }}$ is the first choice of treatment in this setting. HAIC using an implanted port is also recommended for TACE-refractory patients. The regimen for this treatment is usually low-dose FP [5-fluorouracil (5FU) + cisplatin] or intraarterial 5FU infusion combined with systemic interferon therapy. Sorafenib is also a treatment of choice for TACE-refractory patients with Child-Pugh A liver function. ${ }^{f}$ Resection is sometimes performed even when the number of nodules is $>4$. Furthermore, ablation is sometimes performed in combination with TACE. 'Milan criteria: tumor size $\leq 3 \mathrm{~cm}$ and tumor number $\leq 3$; or solitary tumor $\leq 5 \mathrm{~cm}$. Even when liver function is good (Child-Pugh A/B), transplantation is

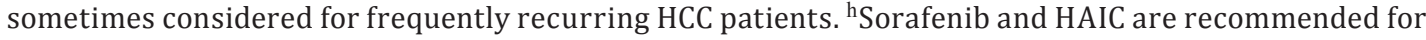
HCC patients with Vp1,2 (minor portal vein invasion) and Vp3 (portal invasion at the 1st portal branch). Sorafenib is not recommended for HCC patients with Vp4 (portal invasion the main portal branch), whereas HAIC is recommended for such patients with tumor thrombus in the main portal branch. ${ }^{i}$ Resection and TACE are frequently performed when portal invasion is minimum, such as Vp1 (portal invasion at the 3rd or more peripheral portal branch) or Vp2 (portal invasion at the 2nd portal branch). ${ }^{\text {LLocal ab- }}$ lation therapy or subsegmental TACE is performed even for Child-Pugh C patients when transplantation is not indicated when there is no hepatic encephalopathy, no uncontrollable ascites, and a low bilirubin level $(<3.0 \mathrm{mg} / \mathrm{dl})$. Although it is a well-accepted treatment in the routine clinical setting, there is no evidence of its survival benefit in Child-Pugh $C$ patients. A prospective study is necessary to clarify this issue. Even in Child-Pugh A/B patients, transplantation is sometimes performed for relatively younger patients with frequently or early recurring HCC after curative treatments.

Modified with permission from Kudo M et al. [19]. 
3 ) If there are $\geq 4$ nodules, the Japanese algorithm recommends that potentially curative therapy with resection and ablation be attempted if possible. Conversely, if there are large nodules or multiple nodules in both hepatic lobes, it recommends HAIC.

4) The European and American guidelines consider sorafenib to be the only treatment indicated for cases involving vascular invasion, whereas the Japanese guidelines also recommend TACE or resection in the case of minor vascular invasion (Vp1 or Vp2) and HAIC in the case of major vascular invasion.

5) Like the European and American algorithms, the Japanese algorithms consider transplantation to be the first-line treatment for HCC patients with Child-Pugh C cirrhosis who meet the Milan criteria. However, the Japanese algorithms completely differ in that it recommends TACE or ablation when transplantation is not possible (e.g., due to donor shortage).

In conclusion, when we compare the Japanese surveillance and diagnostic algorithm and treatment algorithm against the European and American algorithms in detail, the Japanese clinical practice guidelines can be clearly differentiated from the European and American guidelines (and by extension, the management of HCC in these regions can be differentiated), and the Japanese guidelines (and routine diagnosis and treatment of HCC in Japan) can be seen to be different and superior in many aspects.

\section{References}

1 Bruix J, Sherman M, American Association for the Study of Liver Diseases: Management of hepatocellular carcinoma: an update. Hepatology 2011;53:1020-1022.

2 European Association for the Study of the Liver, European Organisation for Research and Treatment of Cancer: EASL-EORTC clinical practice guidelines: management of hepatocellular carcinoma. J Hepatol 2012;56:908-943.

3 Group formed to establish "Guidelines for evidence-based clinical practice for the treatment of liver cancer." Clinical practice guidelines for hepatocellular carcinoma. Tokyo, Kanehara, 2005 (in Japanese).

4 Makuuchi M, Kokudo N, Arii S, Futagawa S, Kaneko S, Kawasaki S, Matsuyama Y, Okazaki M, Okita K, Omata M, Saida Y, Takayama T, Yamaoka Y: Development of evidence-based clinical guidelines for the diagnosis and treatment of hepatocellular carcinoma in Japan. Hepatol Res 2008;38:37-51.

5 Japan Society of Hepatology: Clinical practice guidelines for hepatocellular carcinoma (2013 version). Kanehara, Tokyo, Japan 2013.

6 Kokudo N, Hasegawa K, Akahane M, Igaki H, Izumi N, Ichida T, Uemoto S, Kaneko S, Kawasaki S, Ku Y, Kudo M, Kubo S, Takayama T, Tateishi R, Fukuda T, Matsui O, Matsuyama Y, Murakami T, Arii S, Okazaki M, Makuuchi M: Evidence-based clinical practice guidelines for hepatocellular carcinoma: the Japan Society of Hepatology 2013 update (3rd JSH-HCC Guidelines). Hepatol Res 2015 (in press).

7 Japan Society of Hepatology: Management of Hepatocellular Carcinoma in Japan: Consensus-based Clinical Practice Manual, second edition, Igaku-Shoin, Tokyo, 2010 (in Japanese).

8 Kudo M, Izumi N, Kokudo N, Matsui O, Sakamoto M, Nakashima O, Kojiro M, Makuuchi M, HCC Expert Panel of Japan Society of Hepatology: Management of hepatocellular carcinoma in Japan: Consensus-Based Clinical Practice Guidelines proposed by the Japan Society of Hepatology (JSH) 2010 updated version. Dig Dis 2011;29:339-364.

9 Kudo M: Alpha-fetoprotein-L3: useful or useless for hepatocellular carcinoma? Liver Cancer 2013;2:151152.

10 Song P, Gao J, Inagaki Y, Kokudo N, Hasegawa K, Sugawara Y, Tang W: Biomarkers: evaluation of screening for and early diagnosis of hepatocellular carcinoma in Japan and China. Liver Cancer 2013;2:31-39.

11 Ichikawa T, Sano K, Morisaka H: Diagnosis of pathologically early HCC with EOB-MRI: experiences and current consensus. Liver Cancer 2014;3:97-107.

12 Mise Y, Sakamoto Y, Ishizawa T, Kaneko J, Aoki T, Hasegawa K, Sugawara Y, Kokudo N: A worldwide survey of the current daily practice in liver surgery. Liver Cancer 2013;2:55-66.

13 Lin SM: Local ablation for hepatocellular carcinoma in Taiwan. Liver Cancer 2013;2:73-83.

14 Chan SC: Liver transplantation for hepatocellular carcinoma. Liver Cancer 2013;2:338-344.

15 Raoul JL, Gilabert M, Piana G: How to define transarterial chemoembolization failure or refractoriness: a European perspective. Liver Cancer 2014;3:119-124.

16 Kudo M: Biomarkers and personalized sorafenib therapy. Liver Cancer 2014;3:399-404.

17 Kudo M: Treatment of advanced hepatocellular carcinoma with emphasis on hepatic arterial infusion chemotherapy and molecular targeted therapy. Liver Cancer 2012;1:62-70.

18 Kudo M: Early hepatocellular carcinoma: definition and diagnosis. Liver Cancer 2013;2:69-72. 
19 Kudo M, Matsui O, Izumi N, Iijima H, Kadoya M, Imai Y, Okusaka T, Miyayama S, Tsuchiya K, Ueshima K, Hiraoka A, Ikeda M, Ogasawara S, Yamashita T, Minami T, Yamakado K, Liver Cancer Study Group of Japan: JSH Consensus-based Clinical Practice Guideline for the Management of Hepatocellular Carcinoma: 2014 Update by the Liver Cancer Study Group of Japan. Liver Cancer 2014;3:458-468.

20 Kim YK, Kim CS, Chung GH, Han YM, Lee SY, Jin GY, Lee JM: Radiofrequency ablation of hepatocellular carcinoma in patients with decompensated cirrhosis: evaluation of therapeutic efficacy and safety. AJR Am J Roentgenol 2006;186(Suppl):S261-S268.

21 Kudo M, Osaki Y, Matsunaga T, Kasugai H, Oka H, Seki T: Hepatocellular carcinoma in Child-Pugh C cirrhosis: prognostic factors and survival benefit of nontransplant treatments. Dig Dis 2013;31:490-498.

22 Nishikawa H, Kita R, Kimura T, Ohara Y, Takeda H, Sakamoto A, Saito S, Nishijima N, Nasu A, Komekado H, Osaki Y: Clinical efficacy of non-transplant therapies in patients with hepatocellular carcinoma with ChildPugh C liver cirrhosis. Anticancer Res 2014;34:3039-3044.

23 Wakuta A, Nouso K, Kariyama K, Nishimura M, Kishida M, Wada N, Mizushima T, Higashi T, Tanimoto M: Radiofrequency ablation for the treatment of hepatocellular carcinoma with decompensated cirrhosis. Oncology 2011;81:39-44.

24 Nouso K, Ito Y, Kuwaki K, Kobayashi Y, Nakamura S, Ohashi Y, Yamamoto K: Prognostic factors and treatment effects for hepatocellular carcinoma in Child C cirrhosis. Br J Cancer 2008;98:1161-1165.

25 Nouso K, Kokudo N, Tanaka M, Kuromatsu R, Nishikawa H, Toyoda H, Oishi N, Kuwaki K, Kusanaga M, Sakaguchi T, Morise Z, Kitai S, Kudo M: Treatment of hepatocellular carcinoma with Child-Pugh C cirrhosis. Oncology 2014;87(Suppl 1):99-103. 